\title{
Ranking Abstraction as Companion to Predicate Abstraction ${ }^{\star}$
}

\author{
Ittai Balaban ${ }^{1}$, Amir Pnueli $^{1,2}$, and Lenore D. Zuck ${ }^{3}$ \\ ${ }^{1}$ New York University, New York \\ \{balaban, amir\}acs.nyu.edu \\ ${ }^{2}$ Weizmann Institute of Science \\ ${ }^{3}$ University of Illinois at Chicago \\ lenoredcs.uic.edu
}

\begin{abstract}
Predicate abstraction has become one of the most successful methodologies for proving safety properties of programs. Recently, several abstraction methodologies have been proposed for proving liveness properties. This paper studies "ranking abstraction" where a program is augmented by a nonconstraining progress monitor, and further abstracted by predicate-abstraction, to allow for automatic verification of progress properties. Unlike most liveness methodologies, the augmentation does not require a complete ranking function that is expected to decrease with each step. Rather, the inputs are component rankings from which a complete ranking function may be formed.

The premise of the paper is an analogy between the methods of ranking abstraction and predicate abstraction, one ingredient of which is refinement: When predicate abstraction fails, one can refine it. When ranking abstraction fails, one must determine whether the predicate abstraction, or the ranking abstraction, need be refined. The paper presents strategies for determining which case is at hand.

The other part of the analogy is that of automatically deriving deductive proof constructs: Predicate abstraction is often used to derive program invariants for proving safety properties as a boolean combination of the given predicates. Deductive proof of progress properties requires well-founded ranking functions instead of invariants. We show how to obtain concrete global ranking functions from abstract programs.

We demonstrate the various methods on examples with nested loops, including a bubble sort algorithm on linked lists.
\end{abstract}

\section{Introduction}

Predicate abstraction has become one of the most successful methodologies for proving safety properties of programs. However, with no extension it cannot be used to verify general liveness properties. In this paper, we present a framework, based on predicate abstraction and ranking abstraction, for verification of both safety and progress properties. Ranking abstraction, introduced in [7], is based on an augmentation of the concrete program. The augmentation is parameterized by a set of well founded ranking

\footnotetext{
* This research was supported in part by NSF grant CCR-0205571, ONR grant N00014-99-10131, and Israel Science Foundation grant 106/02-1.
} 
functions. Based on these, new compassion (strong fairness) requirements as well as transitions are generated, all of which are synchronously composed with the program in a non-constraining manner. Unlike most methodologies, the ranking functions are not expected to decrease with each transition of the program. Rather, a further step of state abstraction is performed such that, coupled with the new compassion, it serves to construct a complete ranking function.

The basic premise presented in this paper is that there is a duality between the activities that lead to verification of safety properties via predicate abstraction, and those that lead to verification of progress properties via ranking abstraction. This duality is expressed through the following components:

- The initial abstraction. Heuristics are applied to choose either an initial set of predicates, or a set of core well founded ranking functions.

- Refinement. A too-coarse initial abstraction leads to spurious abstract counterexamples. Depending on the character of the counterexample, either a predicate, or a ranking, refinement is performed.

- Generation of deductive proof constructs. Predicate abstraction is often used as an automatic method to generate an inductive invariant as a boolean combination of the given predicates. Dually, ranking abstraction can be used to generate a global concrete ranking function that decreases with every step of the program, as a lexicographical combination of the core ranking functions.

We demonstrate the use of ranking refinement in order to prove termination of a canonical program with nested loops and unbounded random assignments, as well as a bubble sort algorithm on unbounded linked lists. Both examples entail the use of additional heuristics in order to synthesize core ranking functions.

The framework, as well as all experiments, have been implemented using the TLV interactive model-checker [1]. The contribution of the paper is as follows: At the informal, philosophical level, it strives to convince the reader that the duality between invariance and progress, present in deductive frameworks, extends to how one approaches automatic verification of each kind of property. More concretely, it suggests a formal framework, based on two specific abstraction methods for proving both safety and progress properties. This includes heuristics for choosing separate refinement methodologies based on the form of counterexamples, and a method for automatically deriving a global well founded program ranking function.

The paper is organized as follows: Section 2 describes the computational model of fair discrete systems as well as predicate and ranking abstractions. Furthermore, it motivates the use of ranking abstraction by demonstrating its value, compared to a typical deductive method. Section 3 formalizes the different notions of abstraction refinement. Section 4 presents a method for extracting a global ranking function from an abstract program. Finally, Section 5 summarizes and concludes.

\section{Related Work}

Dams, Gerth, and Grumberg [6] point out the duality between verification of safety and progress of programs. Like us, they aim to lift this duality to provide tools for proving progress properties, whose functionality is analogous to similar tools used for 
safety. Specifically, they propose a heuristic for discovering ranking functions from a program's text. In contrast, we concentrate on an analogy with predicate abstraction, a particular method for safety. Our approach is broader, however, in that we suggest a general framework for safety and progress properties where each of the activities in a verification process has an instantiation with respect to each of the dualities.

In [10] Podelski and Rybalchenko present a method for synthesis of linear ranking functions. The method is complete for unnested loops, and is embedded successfully in a broader framework for proving liveness properties [9].

The topic of refinement of state abstraction, specifically predicate abstraction, has been widely studied. A number of existing works in this area are [5]3], and [4].

\section{The Formal Framework}

In this section we present our computational model, as well as the methods of predicate abstraction and ranking abstraction.

\subsection{Fair Discrete Systems}

As our computational model, we take a fair discrete system (FDS) $S=\langle V, \Theta, \rho, \mathcal{J}, \mathcal{C}\rangle$, where

- $V$ - A set of system variables. A state of $S$ provides a type-consistent interpretation of the variables $V$. For a state $s$ and a system variable $v \in V$, we denote by $s[v]$ the value assigned to $v$ by the state $s$. Let $\Sigma$ denote the set of all states over $V$.

- $\Theta$ - The initial condition: An assertion (state formula) characterizing the initial states.

- $\rho\left(V, V^{\prime}\right)$ - The transition relation: An assertion, relating the values $V$ of the variables in state $s \in \Sigma$ to the values $V^{\prime}$ in an $S$-successor state $s^{\prime} \in \Sigma$.

- $\mathcal{J}$ - A set of justice (weak fairness) requirements (assertions); A computation must include infinitely many states satisfying each of the justice requirements.

- $\mathcal{C}$ - A set of compassion (strong fairness) requirements: Each compassion requirement is a pair $\langle p, q\rangle$ of state assertions; A computation should include either only finitely many $p$-states, or infinitely many $q$-states.

For an assertion $\psi$, we say that $s \in \Sigma$ is a $\psi$-state if $s=\psi$.

A computation of an FDS $S$ is an infinite sequence of states $\sigma: s_{0}, s_{1}, s_{2}, \ldots$, satisfying the requirements:

- Initiality $-s_{0}$ is initial, i.e., $s_{0}=\Theta$.

- Consecution - For each $\ell=0,1, \ldots$, the state $s_{\ell+1}$ is an $S$-successor of $s_{\ell}$. That is, $\left\langle s_{\ell}, s_{\ell+1}\right\rangle \models \rho\left(V, V^{\prime}\right)$ where, for each $v \in V$, we interpret $v$ as $s_{\ell}[v]$ and $v^{\prime}$ as $s_{\ell+1}[v]$.

- Justice - for every $J \in \mathcal{J}, \sigma$ contains infinitely many occurrences of $J$-states.

- Compassion - for every $\langle p, q\rangle \in \mathcal{C}$, either $\sigma$ contains only finitely many occurrences of $p$-states, or $\sigma$ contains infinitely many occurrences of $q$-states. 


\subsection{Predicate Abstraction}

The material here is a summary of [7] and [2]. We fix an FDS $S=\langle V, \Theta, \rho, \mathcal{J}, \mathcal{C}\rangle$ whose set of states is $\Sigma$. A predicate abstraction is a mapping $\alpha: \Sigma \rightarrow\{0,1\}^{n}$ for some positive $n$. The set of tuples $\{0,1\}^{n}$ is referred to as the set of abstract states. We focus on abstractions that can be represented by a set of equations of the form $\left\{u_{i}=P_{i}(V) \mid i=1, \ldots, n\right\}$, where the $P_{i}$ 's are assertions over the concrete variables $V$, to which we refer as predicates, and $U=\left\{u_{1}, \ldots, u_{n}\right\}$ is the set of boolean abstract variables. The mapping $\alpha$ can also be expressed more succinctly by:

$$
U=\mathcal{P}(V)
$$

For an assertion $p(V)$, we define its abstraction by:

$$
\alpha(p): \quad \exists V \cdot(U=\mathcal{P}(V) \wedge p(V))
$$

The semantics of $\alpha(p)$ is $\|\alpha(p)\|=\{\alpha(s) \mid s \in\|p\|\}$. Note that $\|\alpha(p)\|$ is, in general, an over-approximation - an abstract state is in $\|\alpha(p)\|$ iff there exists some concrete $p$-state that is abstracted into it. An assertion $p\left(V, V^{\prime}\right)$ over both primed and unprimed variables is abstracted by:

$$
\alpha(p): \quad \exists V, V^{\prime} .\left(U=\mathcal{P}(V) \wedge U^{\prime}=\mathcal{P}\left(V^{\prime}\right) \wedge p\left(V, V^{\prime}\right)\right)
$$

The assertion $p$ is said to be precise with respect to the abstraction $\alpha$ if $\|p\|=$ $\alpha^{-1}(\|\alpha(p)\|)$, i.e., if two concrete states are abstracted into the same abstract state, they are either both $p$-states, or they are both $\neg p$-states. For a temporal formula $\psi$ in positive normal form (where negation is applied only to state assertions), $\psi^{\alpha}$ is the formula obtained by replacing every maximal state sub-formula $p$ in $\psi$ by $\alpha(p)$. The formula $\psi$ is said to be precise with respect to $\alpha$ if each of its maximal state sub-formulas are precise with respect to $\alpha$.

In all cases discussed in this paper, the formulae are precise with respect to the relevant abstractions. Hence, we can restrict to the over-approximation semantics.

The abstraction of $S$ by $\alpha$ is the system

$$
S^{\alpha}=\left\langle U, \alpha(\Theta), \alpha(\rho), \bigcup_{J \in \mathcal{J}} \alpha(J), \bigcup_{(p, q) \in \mathcal{C}}(\alpha(p), \alpha(q))\right\rangle
$$

The soundness of predicate abstraction is derived from [7]:

Theorem 1. For a system $S$, abstraction $\alpha$, and a positive normal form temporal formula $\psi$ :

$$
S^{\alpha} \models \psi^{\alpha} \quad \Longrightarrow \quad S \models \psi
$$

Thus, if an abstract system satisfies an abstract property, then the concrete system satisfies the concrete property.

\subsection{Ranking Abstraction}

State abstraction often does not suffice to verify progress properties. We consider ranking abstraction, a method of augmenting the concrete program in a non-constraining 
manner, in order to measure progress of program transitions, with respect to a ranking function. Once a program is augmented, a conventional state abstraction can be used to preserve this notion in the abstract system. This method was introduced in [7].

A ranking function is a function mapping program states to some domain $\mathcal{D}$. A ranking function is well founded if $\mathcal{D}$ is partially ordered by a relation $\succ$, which does not admit an infinitely descending chain of the form $a_{0} \succ a_{1} \succ a_{2} \succ \cdots$. Throughout the rest of the paper we assume all ranking functions to be well founded. The augmentation of system $S$ by a ranking function $\delta$, written $S+\delta$, is the system

$$
S+\delta:\left\langle V \cup\{\operatorname{dec}\}, \Theta, \rho \wedge \rho_{\delta}, \mathcal{J}, \mathcal{C} \cup\{(\operatorname{dec}>0, \operatorname{dec}<0)\}\right\rangle
$$

where $d e c$ is a fresh variable symbol, and the conjunct $\rho_{\delta}$ is defined as

$$
\rho_{\delta}: \operatorname{dec}^{\prime}=\left\{\begin{aligned}
1 & \delta \succ \delta^{\prime} \\
0 & \delta=\delta^{\prime} \\
-1 & \text { otherwise }
\end{aligned}\right.
$$

The well foundedness of $\delta$ is abstracted by the augmentation into the compassion requirement $(d e c>0, d e c<0)$, stating that if $d e c$ is positive infinitely often in a computation, then it must also be negative infinitely often.

Since augmentation does not constrain the behavior of $S$, any property in terms of variables of $S$ is valid over $S$ iff it is valid over $S+\delta$. In order to verify a progress property $\psi$, an augmentation $S+\delta$ is abstracted using a standard state abstraction, as shown in Example1.

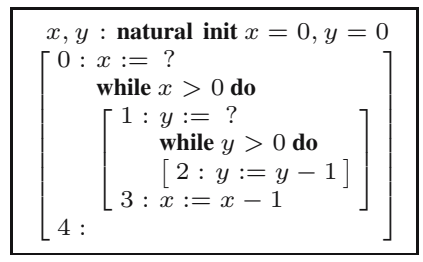

Fig. 1. Program NESTED-LOOPS

Example 1 (Nested Loops). Consider the program NeSTED-Loops in Fig. 1. In the program, the statements $x:=?, y:=$ ? in lines 0 and 1 denote random assignments of arbitrary positive integers to variables $x$ and $y$. An initial attempt to prove termination of this program is to define the ranking function $\delta_{y}=y$. The augmentation $S+\delta_{y}$ is shown in Fig. 2. Note that statements that in the original program assigned to $y$, are now replaced with a simultaneous assignment to both $y$ and the augmentation variable $\operatorname{dec}_{y}$.

While this augmentation is not sufficient to prove program termination, it can be used to show termination of the inner loop, expressed by

$$
(\pi=2) \Longrightarrow \diamond(\pi=3)
$$

where the variable $\pi$ denotes the program counter. As a state abstraction we use

$$
\alpha:(X=(x>0)) \wedge(Y=(y>0)) \wedge\left(\operatorname{Dec}_{y}=\operatorname{dec}_{y}\right)
$$




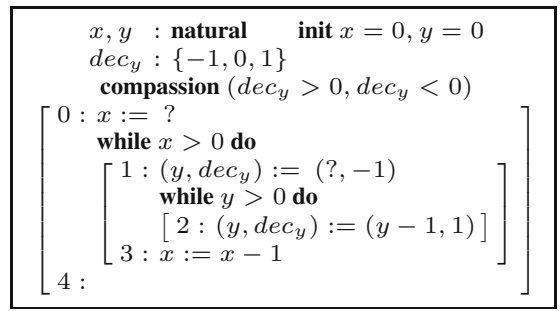

Fig. 2. Program Augmented-Nested-Loops

which results in the abstract program in Fig. 3. Notice that the abstraction has introduced nondeterministic assignments to both $X$ and $Y$ (lines 2 and 3). It is now possible to verify, e.g. by model-checking, the property (1) over the abstract program.

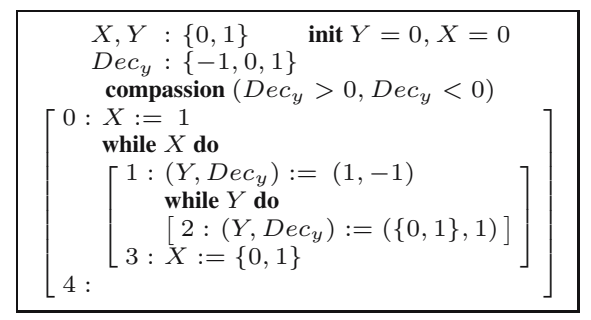

Fig. 3. Program Abstract-Augmented-Nested-Loops

In general, we consider simultaneous augmentation with sets of ranking functions. A ranking is a set of ranking functions. Let $\Delta$ be the ranking $\left\{\delta_{1}, \ldots, \delta_{k}\right\}$. Then the augmentation $S+\Delta$ is the system

$$
S+\Delta: S+\delta_{1}+\cdots+\delta_{k}
$$

Just like the case of predicate abstraction, we lose nothing (except efficiency) by adding potentially redundant rankings. The main advantage here over direct use of ranking functions within deductive verification is that one may contribute as many elementary ranking functions as one wishes. It is then left to a model-checker to sort out their interaction and relevance. To illustrate this, consider a full termination proof of the program NESTED-LOOPS. Due to the unbounded non-determinism of the random assignments, a termination proof needs to use a ranking function ranging over lexicographic tuples, whose elementary ranking components are $\{\pi=0, x, y\}$. With ranking abstraction, however, one need only provide the well founded ranking $\Delta=\{x, y\}$.

\section{Abstraction Refinement}

\subsection{Preliminaries}

In the following sections we refer to finite sequences of consecutive states, known as traces, as well as composite transition relations denoted by traces. Fix a trace 
$\sigma: \sigma_{1} \ldots \sigma_{\ell}$, where the $\sigma_{i}$ are program states. An alternate representation of $\sigma$ is as the sequence of transitions $\rho_{1} \ldots \rho_{\ell-1}$, with $\rho_{i}$ defined as $\sigma_{i} \wedge \sigma_{i+1}^{\prime}$ for each $i<\ell$.

A composition of two transition relations $\rho_{1}$ and $\rho_{2}$, denoted by $\rho_{1} \circ \rho_{2}$, is defined as $\exists V^{\prime} . \rho_{1}\left(V, V^{\prime}\right) \wedge \rho_{2}\left(V^{\prime}, V^{\prime \prime}\right)$. The transition relation denoted by the trace $\sigma$, written $\rho_{\sigma}$, is the composition of its transition relations, given by $\rho_{\sigma}: \rho_{1} \circ \ldots \circ \rho_{\ell-1}$.

A cycle is a trace of the form $\sigma_{1} \ldots \sigma_{\ell} \sigma_{1}$. In the following sections we discuss both cycles and control flow loops, the difference being that while in a cycle the initial and last states are identical, a control flow loop is a trace where the initial and last states assign identical values to the program counter $\pi$, but not necessarily to other variables.

\subsection{Refinement}

The process of proving or refuting a progress property $\psi$ over a program begins with a user-provided initial ranking $\Delta$ and an abstraction $\alpha$. Following [11], the initial predicate abstraction is chosen as follows: Let $\mathcal{P}$ be the (finite) set of atomic state formulas occurring in $\rho, \Theta, \mathcal{J}, \mathcal{C}$ and the concrete formula $\psi$ that refer to non-control and nonprimed variables. Then the abstraction $\alpha$ is the set of equations $\left\{B_{p}=p: p \in \mathcal{P}\right\}$.

The formula $\psi^{\alpha}$ is then model-checked over $(S+\Delta)^{\alpha}$. If $\psi^{\alpha}$ is valid then we can conclude that $S \models \psi$. Otherwise, a counterexample is found, in the form of a computation of $S^{\alpha}$ that does not satisfy $\psi^{\alpha}$. If such a computation exists then a standard model checker will return a counter-example that is finitely represented as a "lasso" - a finite initial trace followed by a cycle. The following scenarios may give rise to a counterexample:

1. The property $\psi$ is not valid: The concrete system indeed has a computation in which a cycle is repeated infinitely

2. The state abstraction is too coarse: The abstract system has a finite trace with no concrete counterpart

3. The concrete system has a finite acyclic trace that cannot be extended to an infinite computation, such that its abstraction contains a cycle.

In case 1 , a valid counterexample to $\psi$ has been found, and we conclude that $\psi$ indeed is not valid over $S$. Cases 2 and 3 represent different forms of infeasibility of the counterexample, and are handled by different means of refinement.

Case 2 represents a typical scenario in state abstraction refinement, in the following way: Although the counterexample may be a lasso, say $\left(\sigma_{1}, \sigma_{2}\right)$, we consider the finite trace $\sigma_{1} \sigma_{2}$ and the fact that it is spurious, i.e., cannot be concretized. This case is therefore handled by refining the abstraction with a new predicate that eliminates the spurious trace. Any one of existing refinement methods may be applied (see, for example, [5|34]).

Case 3 implies the following situation: A cyclic trace is present in the abstract system, the concretization of which is a control-flow loop. Furthermore, the loop is wellfounded, in that there exists no infinite sequence of iterations of the loop. To preserve this property in the abstract program, we search for a ranking function that proves the loop's well-foundedness. Formally, if $\rho_{\sigma}$ is the loop transition relation, then our goal is to find a ranking function $\delta$ over a domain $(\mathcal{D}, \succ)$ such that $\rho_{\sigma} \rightarrow \delta \succ \delta^{\prime}$. A number of methods have been proposed to synthesize such functions, among them in [10,6]. In 
Subsection 3.3 we present an additional heuristic for the domain of unbounded linked lists.

Cases 2 and 3 both result in a refined abstraction and a refined ranking, respectively. Therefore, the verification process continues with a new iteration of augmentation and abstraction. The process repeats until success $-\psi$ is proven to be valid or invalid - or the refinement reaches a fixpoint, in which case the process fails.

Example 2 (Termination of NESTED-LOOPS). Recall the program NESTED-LOOPS in Fig. 1 for which we wish to prove termination, expressed as $(\pi=0) \Longrightarrow \diamond(\pi=4)$. We begin with the initial abstraction and ranking used in Example1.

$$
\begin{aligned}
& \alpha:(X=(x>0)) \wedge(Y=(y>0)) \wedge\left(\text { Dec }_{y}=\operatorname{dec}_{y}\right) \\
& \Delta:\left\{\delta_{1}=y\right\}
\end{aligned}
$$

An initial iteration of abstraction and model-checking results in an abstract lasso counterexample consisting of the prefix

$$
\left\langle\Pi=0, X, \neg Y, D e c_{y}=0\right\rangle
$$

and the cycle

$$
\begin{aligned}
& \left\langle\Pi=1, X, \neg Y, \operatorname{Dec}_{y}=0\right\rangle \\
& \left\langle\Pi=2, X, \quad Y, \operatorname{Dec}_{y}=-1\right\rangle \\
& \left\langle\Pi=3, X, \neg Y, \operatorname{Dec}_{y}=1\right\rangle
\end{aligned}
$$

with $\Pi$ denoting the program counter in the abstract program. The lasso can be concretized, as shown by the concrete trace

$$
\begin{aligned}
& s_{1}:\left\langle\pi=0, x=0, y=0, \text { dec }_{y}=0\right\rangle \\
& s_{2}:\left\langle\pi=1, x=4, y=0, \operatorname{dec}_{y}=0\right\rangle \\
& s_{3}:\left\langle\pi=2, x=4, y=1, \operatorname{dec}_{y}=-1\right\rangle \\
& s_{4}:\left\langle\pi=3, x=4, y=0, \text { dec }_{y}=1\right\rangle \\
& s_{5}:\left\langle\pi=1, x=3, y=0, \text { dec }_{y}=0\right\rangle
\end{aligned}
$$

Therefore the counterexample falls under Case 3 above, and we examine a concretization of the cyclic portion of the lasso, i.e., the subtrace $\sigma: s_{2} \ldots s_{5}$. Forming its transition relation $\rho_{\sigma}$, we now examine its implication on transitions of the program variables, in the hope of proving well-foundedness. Specifically, we discover that the constraint $x>x^{\prime}$ is implied by $\rho_{\sigma}$. This suggests refining $\Delta$ with the ranking function $\delta_{2}=x$, to form $\Delta^{\prime}:\left\{\delta_{1}=y, \delta_{2}=x\right\}$. At this point, the abstraction of $S+\Delta^{\prime}$ by $\alpha$ is sufficient to verify the termination property.

\subsection{Synthesizing Elementary Ranking Functions}

A number of methods have been suggested for synthesis of ranking functions. In our examples we have used the general heuristic of searching for simple linear constraints implied by the transition relation of a control-flow loop ([10] provides a less naïve method for doing this. Indeed, their method is complete). For example, given a set of variables $V$ and the transition relation $\rho_{\sigma}$ of a loop, we check validity of implications 
such as $\rho_{\sigma} \rightarrow v>v^{\prime}$, for each $v \in V$. As demonstrated, this has been sufficient in dealing with the NESTED-LOOPS program.

We have used a variant of this heuristic to deal with programs that manipulate unbounded pointer structures. One such program is BUBBLE SORT, shown in Fig. 4. This is a parametrized system with $H$ denoting the maximal size of a singly-linked pointer structure (or heap). The heap itself is represented by the array $N x t$. In addition there are a number of pointer variables, such as $x$ and $y$, that are also parametrized by $H$.

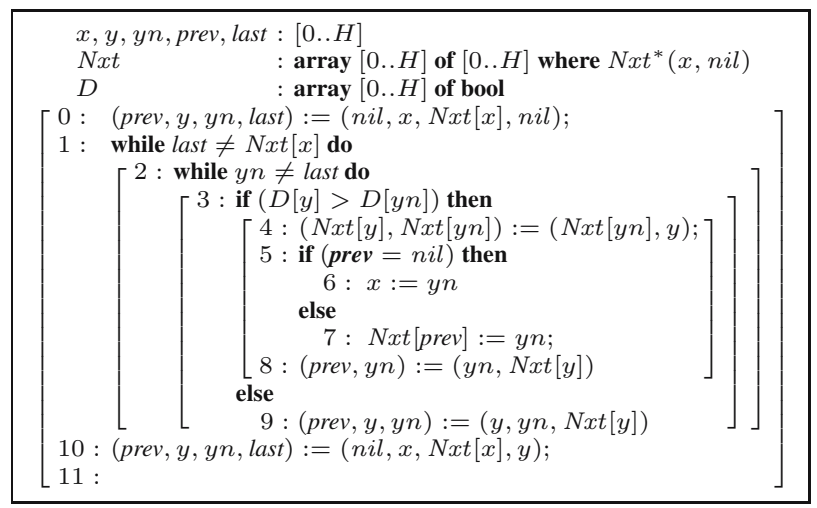

Fig. 4. Program Bubble SoRT

In order to synthesize a ranking function for BUBBLE SORT and similar programs, our strategy is to seek constraints on graph reachability. One such form of constraint is

$$
\rho_{\sigma} \rightarrow\left(\operatorname{reach}\left(v, v^{\prime}\right) \wedge v \neq v^{\prime}\right)
$$

where $\rho_{\sigma}$ is a loop transition relation and $v$ is a pointer variable. Under the assumption that a singly-linked list emanating from $v$ is acyclic, such a constraint suggests the ranking function $\{i \mid \operatorname{reach}(v, i)\}$ over the domain $\left(2^{\mathbb{N}}, \supset\right)$. Indeed, while proving termination of BUBBLE SORT, one of the functions discovered automatically by refinement was $\{i \mid \operatorname{reach}(y n, i)\}$, a function that serves to prove termination of the nested loop (lines $2 \ldots 9)$.

\section{Extracting Complete Ranking Functions}

This section provides an overview of an algorithm that extracts a ranking function from a program constructed by a joint (ranking and predicate) abstraction. The algorithm manipulates a symbolic (BDD-based) representation of the system, and is an adaptation of the explicit state version in [8].

The algorithm EXTRACT-RANKING, shown in Fig. 5, is based on a partitioning of the control flow graph of an abstract program into strongly connected components. It distinguishes between components that are singletons with no edges, and components with 


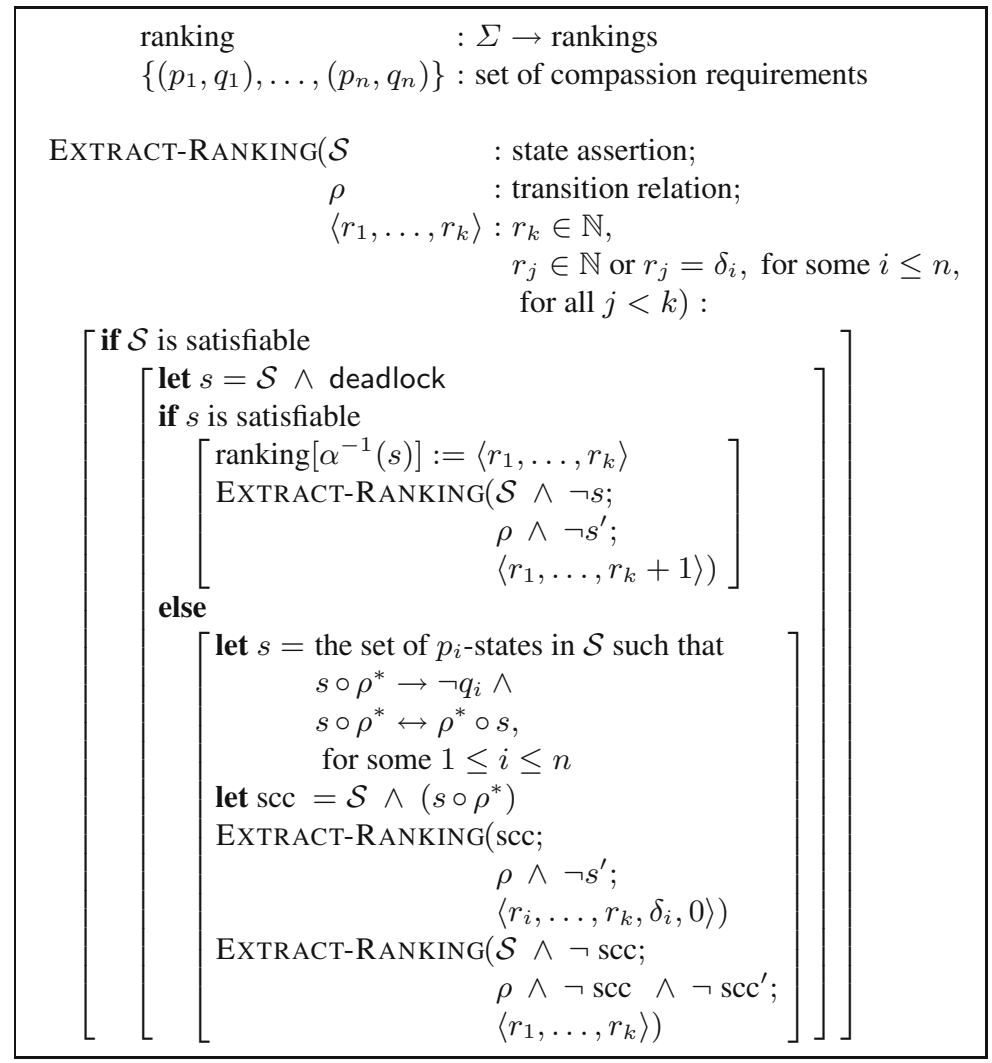

Fig. 5. Ranking Extraction Algorithm. The notations $s \circ \rho^{*}$ and $\rho^{*} \circ s$ denote, respectively, the sets of eventual successors and eventual predecessors of $s$.

at least one transition. The observation is that zero-edge singleton components represent program states that reside outside of any control-flow loop, while other components represent loops. A further observation is that if an always-decreasing ranking function indeed exists, any loop is necessarily unfair, i.e. there exists some fairness requirement that is not satisfied by a run that never escapes the loop. For clarity of presentation we assume that there are no justice requirements, only compassion. We also assume that all compassion requirements have been generated as a result of ranking abstraction, and hence each requirement $\left(p_{i}, q_{i}\right)$ is associated with a component ranking function $\delta_{i}$.

The algorithm iterates over the set of components, singleton or otherwise, while constructing a mapping between state sets and rankings. A ranking is a lexicographic tuple of the form $\left\langle r_{1}, \ldots, r_{k}\right\rangle$, where for all $j<k, r_{j}$ is either a nonnegative integer, or the ranking function $\delta_{i}$ associated with the compassion $\left(p_{i}, q_{i}\right)$. The element $r_{k}$ is always a nonnegative integer.

Initially the algorithm is called with the set of reachable abstraction states, the abstract transition relation, and the tuple $\langle 0\rangle$ as inputs. At every iteration, the algorithm first attempts to prune all deadlock states. It assigns the ranking $\left\langle r_{1}, \ldots, r_{k}+1\right\rangle$ to the concretization of every such state, where $\left\langle r_{1}, \ldots, r_{k}\right\rangle$ is the the ranking assigned 


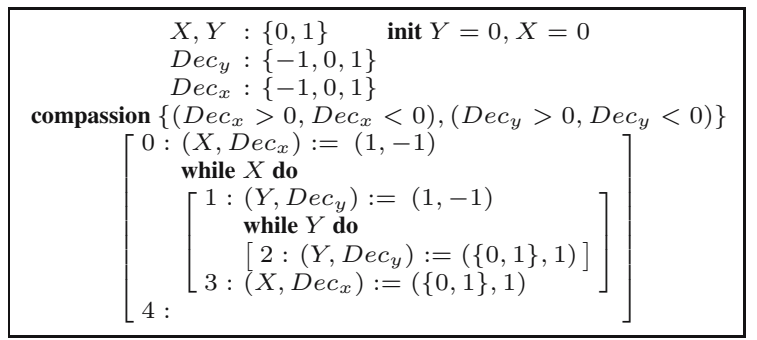

Fig. 6. Abstraction of Program Nested-LOoPS augmented with ranking functions $\delta_{1}=y$ and $\delta_{2}=x$

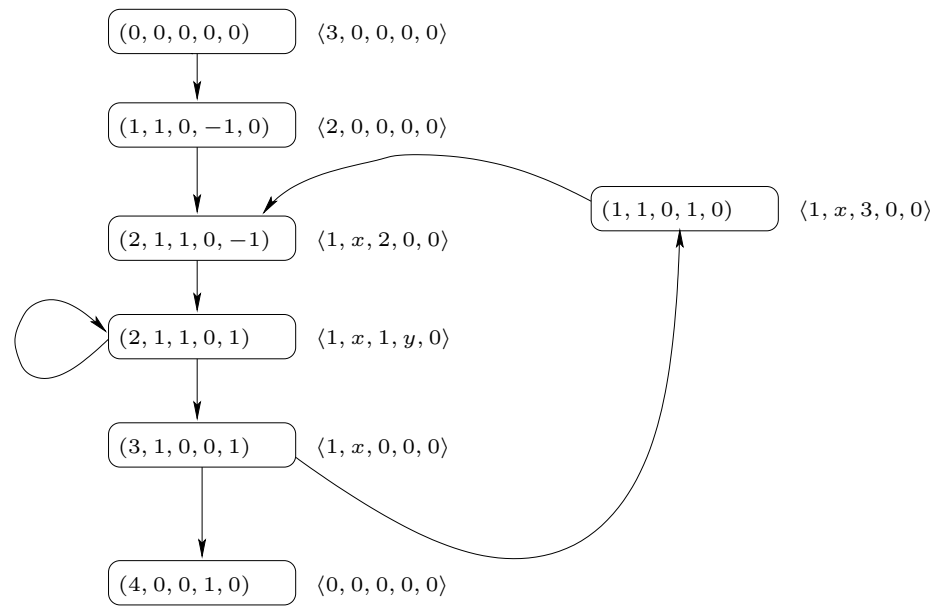

Fig. 7. Abstract Control Flow Graph and Ranking Function of NESTED-Loops. Each tuple $(a, b, c, d, e)$ denotes the abstract program state $\left(\Pi=a, X=b, Y=c, D e c_{x}=d, D e c_{y}=e\right)$.

in the previous iteration. If no deadlock exists, an arbitrary component is picked that has no outgoing edge and violates a compassion requirement $\left(p_{i}, q_{i}\right)$, for some $i$. That is, the component contains one or more $p_{i}$-states, but no $q_{i}$-state. At this point the outgoing edges of the $p_{i}$-states are pruned from the graph. The algorithm is then applied recursively on what remains of the component, with the input ranking of $\left\langle r_{1}, \ldots, r_{k}+1, \delta_{i}, 0\right\rangle$, where $\left\langle r_{1}, \ldots, r_{k}\right\rangle$ is the ranking assigned in the previous iteration. This means that all rankings assigned by the recursive call will contain the prefix $\left\langle r_{1}, \ldots, r_{k}+1, \delta_{i}\right\rangle$.

Example 3 (Extracting a Ranking Function of NESTED-LOOPS). We illustrate the algorithm by extracting a ranking function for NESTED-LOOPS, given the abstract program shown in Fig. 6. This is a version of the augmented abstract version from Fig. 3, after refinement with the ranking function $\delta_{2}=x$.

Fig. 7 shows the control flow graph of the abstract program, with a ranking associated with each state. The interpretation of this diagram is a ranking function defined as follows: For any concrete state $s$ that abstracts to a graph state $\alpha(s)$, the rank of $s$ is the lexicographic tuple associated with $\alpha(s)$. 
Space limitations prevent us from presenting a proof that the resulting ranking function is in fact always-decreasing.

\section{Conclusion}

The work in this paper is a direct continuation of [2], where a framework was presented for automatic computation of predicate and ranking abstractions, with a specific application to the domain of unbounded pointer structures (aka Shape Analysis). That framework requires all predicates and component ranking functions to be provided by the user. Here we have extended it with dual means of refinement for both types of abstraction.

We have shown two heuristics for synthesizing component ranking functions, one for a linear domain and another for a domain of unbounded pointer structures. These have been surprisingly effective in proving termination of a number of example programs. In the near future we plan to explore richer heuristics in the domain of shape analysis.

\section{References}

1. A. Pnueli and E. Shahar. A platform combining deductive with algorithmic verification. In Rajeev Alur and Thomas A. Henzinger, editors, Proceedings of the Eighth International Conference on Computer Aided Verification CAV, volume 1102, page 184, New Brunswick, NJ, USA, / 1996. Springer Verlag.

2. I. Balaban, A. Pnueli, and L. D. Zuck. Shape analysis by predicate abstraction. In VMCAI'2005: Verification, Model Checking, and Abstraction Interpretation, pages 164-180, 2005.

3. T. Ball, A. Podelski, and S. K. Rajamani. Relative completeness of abstraction refinement for software model checking. In Tools and Algorithms for Construction and Analysis of Systems, pages $158-172,2002$.

4. T. Ball and S. K. Rajamani. Automatically validating temporal safety properties of interfaces. Lecture Notes in Computer Science, 2057:103+, 2001.

5. E. M. Clarke, O. Grumberg, S. Jha, Y. Lu, and H. Veith. Counterexample-guided abstraction refinement. In Computer Aided Verification, pages 154-169, 2000.

6. D. Dams, R. Gerth, and O. Grumberg. A heuristic for the automatic generation of ranking functions. In G. Gopalakrishnan, editor, Workshop on Advances in Verification, pages 1-8, 2000.

7. Y. Kesten and A. Pnueli. Verification by augmented finitary abstraction. Information and Computation, 163(1):203-243, 2000.

8. O. Lichtenstein and A. Pnueli. Checking that finite-state concurrent programs satisfy their linear specification. In Proc. 12th ACM Symp. Princ. of Prog. Lang., pages 97-107, 1985.

9. A. Podelski and A. Rybalchenko. Software model checking of liveness properties via transition invariants. Research Report MPI-I-2003-2-004, Max-Planck-Institut für Informatik, Stuhlsatzenhausweg 85, 66123 Saarbrücken, Germany, December 2003.

10. A. Podelski and A. Rybalchenko. A complete method for the synthesis of linear ranking functions. In Verification, Model Checking, and Abstract Interpretation, pages 239-251, 2004.

11. S. Graf and H. Saïdi. Construction of abstract state graphs with PVS. In O. Grumberg, editor, Proc. 9th International Conference on Computer Aided Verification (CAV'97), volume 1254, pages 72-83. Springer Verlag, 1997. 